I.

Aus dem pharmakol. Institut Breslau (Direktor: Geh.-Rat Prof. Dr. Pohl).

\title{
Ueber die Wirkung ätzender Ester (unter Berücksichtigung der Gaskampfstoffe).
}

Von

Curt Wachtel.

(Mit 4 Kurven in 'Text.)

Ausgehend von der Absicht, eine im hiesigen pharmakologischen Institut im Jahre 1915 begonnene Versuchsreihe zur Erklärung der Wirkung des Schwefelsäuredimethylesters zu vervollständigen, ergab sich die Notwendigkeit eine Reihe von Versuchen mit diesem Stoffe anzustellen, die im folgenden zunächst angeführt werden sollen.

Um die Beteiligung der einzelnen Komponenten des Dimethylsulfats an seiner Wirksamkeit festzustellen, wurden sodann Versuche mit anderen neutralen Estern der aliphatischen Reihe, insbesondere mit den Estern der Phosphorsäure, Oxalsäure und Kohlensäure vorgenommen, die anschliessend beschrieben werden.

Den Schluss der Mitteilung bilden pathologisch-anatomische Beobachtungen, die Verfasser gelegentlich seiner Tätigkeit in der pharmakologischen Abteilung des Kaiser Wilhelm-Instituts ${ }^{1}$ ) für Physikalische Chemie und Elektrochemie in den Jahren 1916 und 1917 bei der Untersuchung über die Wirkung mehrerer hundert für die Einführung als Gaskampfstoffe in betracht kommender Substanzen gesammelt hat und die zum Teil über das Gebiet der eigentlichen Kriegswissenschaften hinaus für die gewerblichen Vergiftungen Interesse beanspruchen.

1.

Die Theorie der Dimethylsulfatvergiftung ist von Weber ${ }^{2}$ ) entwickelt worden, welcher zu dem Schlusse kommt, dass die Wirkungen des Dimethylsulfats von dem ganzen Molekül der Verbindung und nicht von den abgespaltenen Komponenten desselben, Schwefelsäure und

1) An dieser Stelle sei es mir gestattet, mit ganz besonderem Danke der Zusammenarbeit mit Herrn Prof. Dr. F. Flury zu gedenken, welcher als Leiter der Pharmakologischen Abteilung des Kaiser Wilhelm-Instituts die angeführten Untersnchungen des Verfassers stets in bereitwilligster Weise unterstützte.

2) Arch. f. exper. Path. u. Pharm. 1902, Bd. 47. S. 113. 
Methylalkohol abhängen. Die heftig ätzende Wirkung des Dimethylsulfats an allen Applikationsstellen mit Einschluss der Lungen nach Einatmung der Dämpfe ist nach Weber dem Gesamtmolekül als solchem eigentümlich. Ueber die Ursache der Wirkung bemerkt Weber weiterhin: „Zunächst sind Schwefelsäurester im Organismus nicht leicht zersetzlich, ferner sind die Schwefelsüuremengen, die in Form des Dimethylsulfats in den Magen eingeführt sind, sehr gering. Bei einem Kaninchen hatten $0,1 \mathrm{~g}$ Dimethylsulfat (darin $0,076 \mathrm{~g} \mathrm{SO}_{4}$ ) ausgedehnte Magenätzung zur Folge. Die Säuremenge wäre sicher nicht imstande gewesen, so ausgedehnte Zerstörungen zu veranlassen ...." „Zur weiteren Kontrolle wurde dann noch der Versuch gemacht, durch gleichzeitige Alkalidarreichung, die bei säurevergifteten Tieren so eklatante Heilwirkungen hat, die Vergiftungen zu beeinflussen. Weder die subkutane noch intravenöse Injektion hatten einen Einfluss. Berücksichtigt man noch, dass bei Säurevergiftungen Krämpfe nicht vorkommen, so wird man nach dem Gesagten eine solche durch das Dimethylsulfat sicher ausschliessen können."

Die nunmehr folgenden Versuche legen jedoch eine abweichende Auffassung nahe, indem sie dafür sprechen, dass neben der von Weber mit Recht angenommenen Wirkung des Gesamtmoleküls gleichzeitig auch eine solche durch Säurespaltung statthat. Berücksichtigt man nämlich neben den funktionellen Veränderungen, welche durch die Ester der Mineralsäuren verursacht werden, auch das gesamte anatomische Krankheitsbild, ferner die Tatsache, dass diese Ester in wässriger Lösung sich nachweisbar viel schneller verseifen als bisher bekannt, übersieht man insbesondere die schweren Aetzwirkungen nicht, welche durch das Dimethylsulfat bei der Einatmung und bei direkter Einwirkung auf die Haut entstehen, während analoge Veränderungen durch den Ester der harmlosen Kohlensäure nicht hervorgerufen werden können, so ist nicht einzusehen, wie man die Annahme einer Wirkung durch Säureabspaltung völlig von der Hand weisen kann. Folgende Versuche seien deshalb hier zunächt angeführt:

1. Versuch: Das käufliche Dimethylsulfat, welches eine ölige, unter teilweiser Zersetzung bei etwa $188^{\circ}$ siedende Flüssigkeit darstellt, ist in Wasser schwer löslich. Unter starkem Schütteln gelingt es, einen Kubikzentimeter Dimethylsulfat in $40 \mathrm{ccm}$ destillierten Wassers ohne Erwärmen zur Lösung zu bringen. Die frisch bereitete Lösung reagiert stark sauer und scheidet ununterbrochen, bis zur völligen Zersetzung des Dimethylsulfats, Säure ab. Zur Neutralisation einer derartigen Lösung waren erforderlich für $20 \mathrm{com}$ der $21 / 2$ proz. Lösung:

Unmittelbar nach der Herstellung $36,0 \mathrm{ccm} \mathrm{n} / 10 \mathrm{NaOH}$, nach einstündigem Stehen weitero 3,4 n $/ 10$ n nach 24 Stunden weitere ... 13,4 "n/10" Also im ganzen $52,8 \mathrm{ccm} \mathrm{n} / 10 \mathrm{NaOH}$ entsprechend 0,254 $\mathrm{g}$ Schwefelsäure und 0,33 $\mathrm{g}$ Dimethylsulfat.

Der Versuch zeigt, dass Dimethylsulfat sich mit Wasser immerhin so schnell zersetzt, dass die Möglichkeit der Wirkung durch Säureab- 
Ueber diø Wirkung ätzender Ester (unter Berücksichtigung der Gaskampfstoffe). 3

spaltung im Organismus mit der Verseifungsgeschwindigkeit in wässriger Lösung vereinbar ist.

2. Versuch: Die Wirkung der aus dem Dimethylsulfat in wässriger Lösung abgespaltenen Säure wird auch durch folgenden Versuch demonstriert: $0,5 \mathrm{ccm}$ Dimethylsulfat mit physiologischer Kochsalzlösung auf $10 \mathrm{ccm}$ aufgefüllt, mit Natronlauge schwach alkalisch gemacht, werden zu $10 \mathrm{ccm}$ einer Lösung von Organeiweiss, hergestellt duroh Auflösen von getrockneter Kaninchenleber in physiologischer Kochsalzlösung, hinzugefügt. Die klare Mischung wird darch Phenolphtalein schwach gefärbt, nach wenigen Minuten tritt Entfärbung ein, etwas später Trübung, nach 24 Stunden reichlicher flockiger Niederschlag.

Lässt man in gleichen Mengenverhältnissen Dimethylsulfat auf Pferdeblutserum einwirken, so tritt nach einigen Minuten ebenfalls eine Entfärbung der mit Phenolphthalein vorher schwach gefärbten J.ösung ein, die Ausflockung von Eiweiss bleibt jedoch aus.

Der Versuch zeigt, dass das Organeiweiss ${ }^{1}$ ) gegen die vom Dimethylsulfat abgespaltene Säure überaus empfindlich ist, während das Serumeiweiss in weit höherem Masse gegen die Säurewirkung geschützt ist. Da Dimethylsulfat erst bei längerer Digestıon durch die Körperflüssigkeiten zerstört wird, so steht das Auftreten resorptiver Wirkungen fern von der Applikationsstelle mit der Annahme einer lokalen Wirkung durch Säureabspaltung nicht in Widersprach.

Um die Wirkungen des Dimethylsulfats zu beobachten, hat Weber alle Arten der Applikation, auch die intravenöse Injektion, angewendet, seine Beobachtungen beschränkten sich jedoch auf die klinischen Erscheinungen im Vergiftungsbilde. Da die reinste Form der Vergiftung bei intravenöser Injektion zu erwarten war, so wurde diese Art der Applikation in den folgenden Versuchen zur Anwendung gebracht, wobei der Verlauf der Vergiftung durch Aufzeichnung der Blutdrucks- und Atmungskurve kontrolliert wurde.

3. Versuch: Kaninchen von $1800 \mathrm{~g}$. $1 \mathrm{ccm}$ Dimethylsulfat wird unter starkem Schütteln in $20 \mathrm{ccm}$ physiologischer Kochsalzlösung gelöst. Die sauer reagierende Lösung wird unter Zusatz von 2 Tropfen Phenolphthalein neutralisiert. Von dieser Lösung werden dem Kaninchen $5 \mathrm{ccm}$ in die rechte Vena jugularis injiziert. Während der Injektion tritt keine Veränderung von Blutdruck und Atmung ein, kurz vor Ende derselben und im unmittelbaren Anschluss daran Vaguspulse, Verlangsamung und geringe Unregelmässigkeit der Atmung. Nach 4 Minuten beginnende Blutdrucksenkung, nach 5 Minuten Aussetzen der Atmung, Zittern des ganzen Körpers und der Extremitäten, fibrilläre Zuckungen am Maule, Schreien. Nach 10 Minuten erneuter Krampfanfall, weitere Blutdrucksønkung und Unregelmässigkeit der Atmung. Nach 11 Minuten Streckkrampf, Verschwinden der Kornalreflexe, exspiratorischer Atemstillstand.

Die Seltion ergibt keine sichtbaren Veränderungen an den Organen, das Serum reagiert alkalisch und zeigt keine Hämolyse.

Die Kurve zeigt, dass Blutdrucksenkung und Atemlähmung die wesentlichen funktionellen Veränderungen bei der Vergiftung mit Dimethylsulfat darstellen.

1) Vgl. hierzu: J.Po hl, Hofmeister's Beitr. z. chem. Physiol. 1905. Bd.7. S.381. 


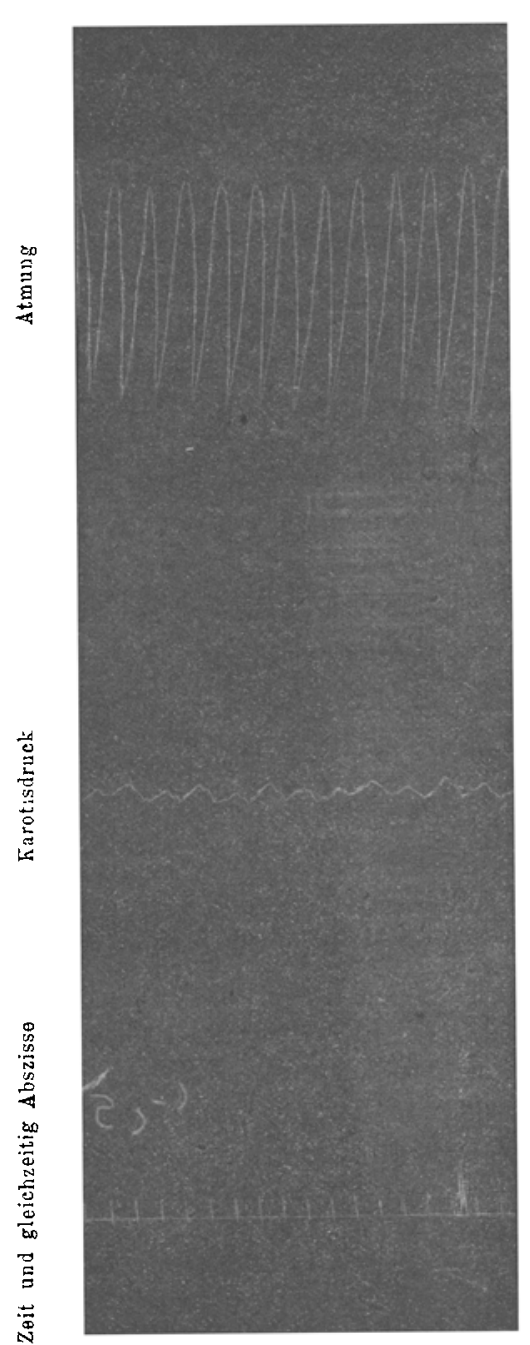

Blutdruck und Atmung bei Beginn des Versuchs (11 thr 25 Min.).

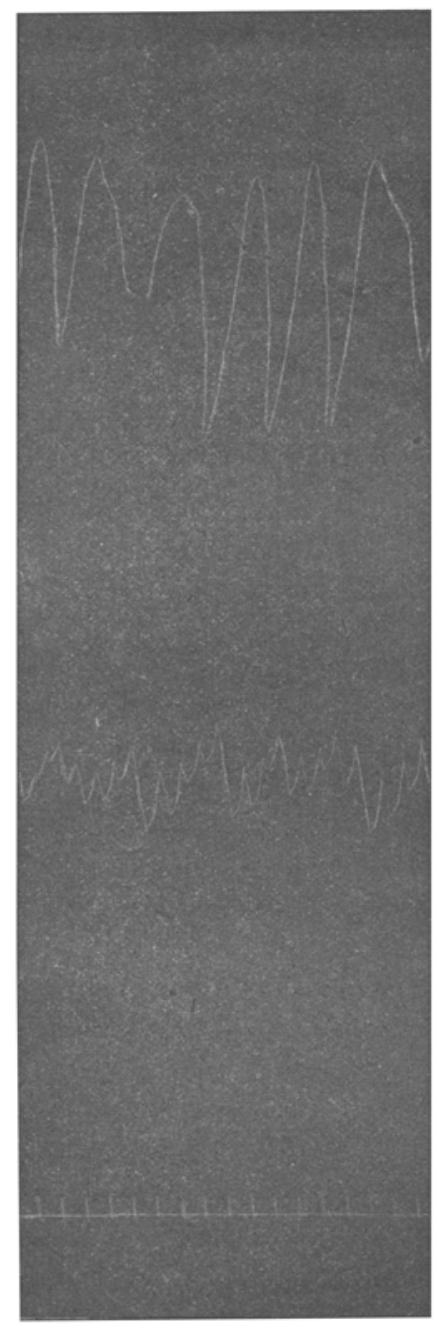

B]ntdruck und Atmungr nach Ende der Injelition von $5 \mathrm{ccm} 5$ proz. Lösung von Dimethylsulfat (11 Uhr 26 Min.). Atmung unregelmässig und verlangsamt. Vaguspulse.

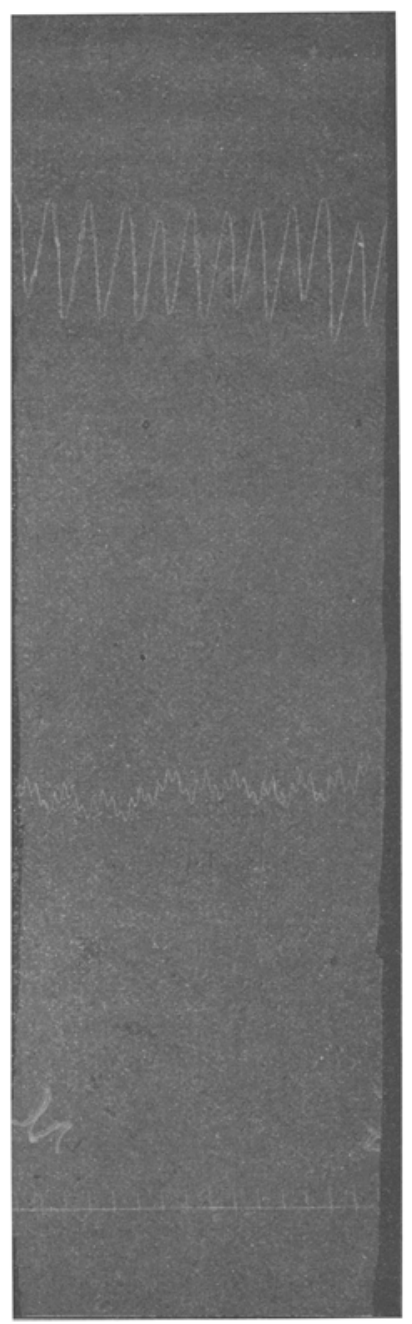

Um 11 Uhr 31 Min. (5 Min. nach der Injektion.)

Um die im vorhergehenden Versuche zutage getretene Wirkung des Nervus vagus auszuschalten, wurde im folgenden Versuche noch einmal ein Kaninchen mit Dimethylsulfat vergiftet, nachdem die Vagi zuvor durch Atropin ausgeschaltet worden waren.

4. Versuch: Kaninchen von $1700 \mathrm{~g}$ erhält intravenös Atropinum sulfuricum $0,002 \mathrm{~g}$ und eine Minute später $5 \mathrm{ccm}$ einer wie in dem vorstehenden Versuche frisch hergestellten und neutralisierten Lösung von Dimethylsulfat. Während der Injektion tritt eine geringe Blutdrucksenkung ein, die nach einigen Sekunden zur Norm zurückkebrt. Nach 3 Minuten fibrilläre Zuckungen am Maul und gөring $\theta$ Blutdrucksenkung, 
Ueber die Wirkung ätzender Ester (unter Berücksichtigung der Gaskampfstoffe). 5

nach 5 Minuten Schreien und wiederholte Krampfstösse mit fibrillären Zuckungen an Maul ond Extremitäten, die Kornealreflexe sind vorhanden. Nach 10 Mi-

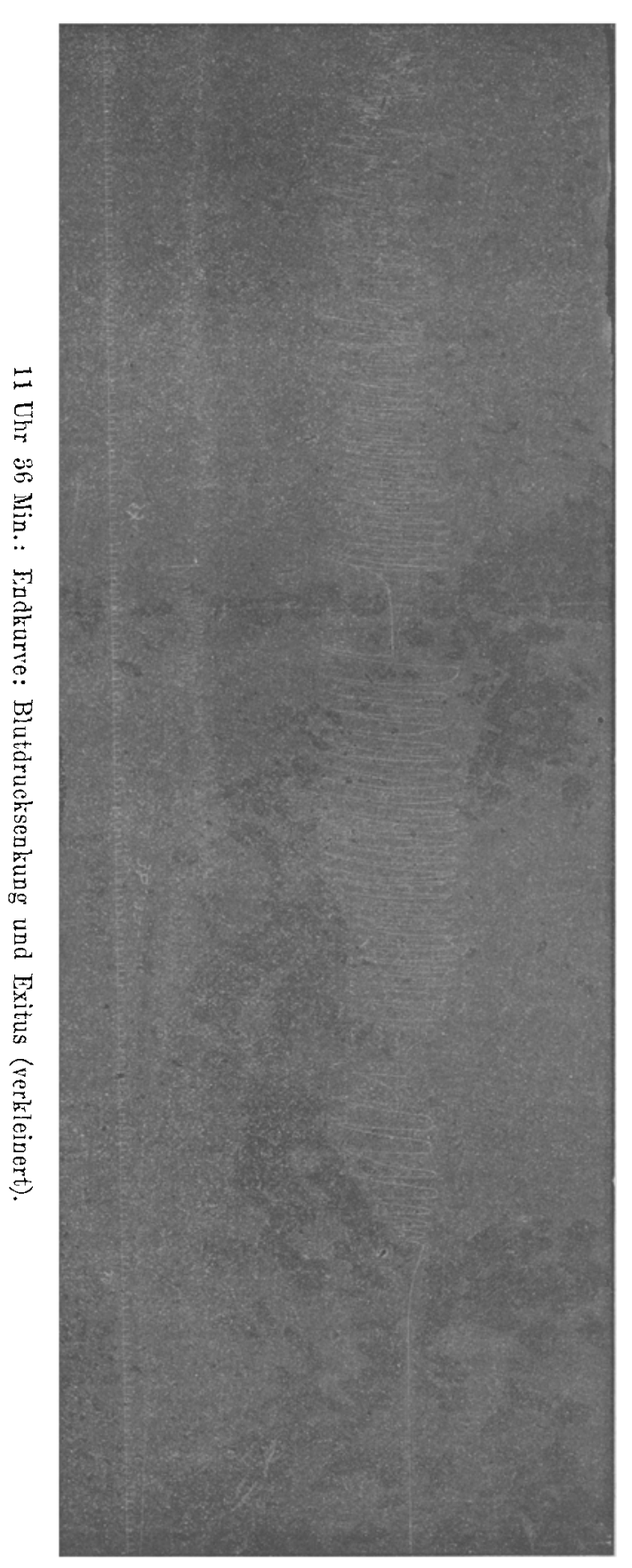
nuten Streckkrämpfe und wiederholte aufeinander folgende Krampfanfälle mit dazwischen liegenden Lähmungsstadien. Nach $15 \mathrm{Mi}$ nuten sind die Kornealreflexe nur noch schwach vorhanden, Parese der Extremitäten, einzelne Sohreie, fibrilläre Zuckungen am Maule. Nach 19 Minuten sind die Kornealreflexe sebr schwach, nach 23 Minuten fehlen sio vollständig, nach 27 Minuten wiederboltes Schreien, der Blutdruck weist inzwischen nach wiederholten Schwankungen eine allmählich zu * nehmendeSenkung auf, das Tier wird nach 65 Minuten abgespannt. Nach $94 \mathrm{Mi}$ nuten Exitus. Die Sektion zeigt keine bemerkenswerten Veränderungen an den F Organen.

Die Vaguspulse sind durch das Atropin aufgehoben worden, im übrigen bestätigt dieser Versuch den vorhergehenden. Da in der Blutdruckkurvedie einzelnen Herzschläge bis zum Schluss gut ausgebildet erscheinen, so handelt es sich hier, zusammengehalton mit dem übrigen Symptomenkomplex, um eine zentral ausgelöste Blutdrucksenkung.

5. Versuch: Um den Verlauf der Dimothylsulfatvergiftung nach sehr. geringen Dosen kennen zu lernen, werden einem Kaninchen von $1100 \mathrm{~g} 0,5 \mathrm{ccm}$ einer wie in den vorhergehenden Versuchen bereiteten und neutralisierten Lösung von Dimethylsulfat in die Randvene 
des rechten Ohres injiziert (ohne Kontrolle von Blatdruck und $\Lambda$ tmung). Das Tier ist nach 2 Stunden etwas matt, Puls und Atmung zeigen keine Besonderheiten. Nach 6 Stunden ist das rechte Ohr ödematös geschwollen, entzündet, hängt schwer herunter, Puls und Atmung zeigen keine Veränderungen. In den nächsten drei Tagen bleibt das Befinden unverändert, die Entzündung des Obres wird noch otwas stärker, geringe Gewichtsabnahm $\theta$ von $40 \mathrm{~g}$ am dritten 'Tage.

Am vierten Versuchstage wird erneut $1,0 \mathrm{ccm}$ der Lösung in die linke Ohrvene injiziert. Am nächsten (5.) Tage macht das Tier einen matten Eindruck, die Entzündung des linken Ohres ist etwas geringer als die des rechten. Gewicht $1020 \mathrm{~g}$, am sechsten Tage der gleiche Befund, Gewicht $970 \mathrm{~g}$, am achten Tage Exitus, Gewicht vor dem Tode $900 \mathrm{~g}$. Die Sektion ergibt keine wesentlichen anatomischen Veränderungen an den lebenswichtigen Organen, an den Ohren einige unerbebliche Nekrosen.

Der Versuch zeigt, dass nach geringen Dosen Dimethylsulfat eine Vergiftung von schleichendem Krankheitsverlauf unter beständiger Gewichtsabnahme zum Tode führt, und bestätigt damit die Beobachtungen an Katzen, welche nach der Einatmung sehr geringer Dosen unter analogen Erscheinungen zugrunde gingen ${ }^{1}$ ). Das hier beobachtete Vergiftungsbild entspricht also dem weiter unten beschriebenen dritten Krankheitstypus, welcher charakterisiert ist durch eine allgemeine Gewebsschädigung und eine toxische Kachexie.

6. Versuch: Um zu untersuchen, ob bei der Einwirkung von Wasser auf Dimethylsulfat pharmakologisch wirksame Substanzen entstehen, wurden $10 \mathrm{ccm}$ der in der üblichen Weise bereiteten Lösung 24 Stunden bei Zimmertemperatur stehen gelassen. Die Lösung zeigte saure Reaktion und einen sulfitartigen Geruch. Die Lösung wurde mit Natronlange neutralisiert, so dass sie nunmehr wohl im wesentlichen $\mathrm{Na}_{2} \mathrm{SO}_{4}$ und Methylalkohol enthielt, und in zwei Portionen von je $5 \mathrm{ccm}$ einem Kaninchen von $1700 \mathrm{~g}$ in die rechte Halsvene injiziert.

Der Erfolg bestand in einer vorübergehenden Respirationshemmung, auf welche alsbald Erholung folgte.

7. Versucb: In analoger Weise wurde die Einwirkung von Blutserum auf Dimethylsulfat untersucht. $1,0 \mathrm{ccm}$ Dimethylsulfat und $5 \mathrm{ccm}$ pbysiologische Kochsalzlösung werden mit Natriumkarbonat neutralisiert, auf $10 \mathrm{ccm}$ aufgofüllt, mit $10 \mathrm{ccm}$ Pferdeserum vermischt und 24 Stunden stehen gelassen. Von dieser Lösung erhält Kaninchen $1940 \mathrm{zweimal}$ je $5 \mathrm{ccm}$ in die rechte Halsvene injiziert.

Der Erfolg besteht in einer geringen Blutdrucksenkung und in einer Veränderung der Herztätigkeit, über welche sich jedoch wegen der bierfür nicht ausreichenden Empfindlichkeit der Apparatur nichts Näheres sagen lässt. Nach einer Stunde tritt wieder eine geringe Erhöhung des Blutdrucks ein, die Pulse werden regelmässig, das Kaninchen wird abgebunden.

Nach dem Versuch keine nachweisbaren Veränderungen, das Tier überlebt.

Die beiden vorstehenden Versuche beweisen, dass das Dimethylsulfat - abgesehen von der 'Säureabspaltung - durch die Körperflüssigkeiten in nahezu unwirksame Produkte verwandelt wird.

Die funktionellen Veränderungen, welche durch das Dimethylsulfat hervorgerufen werden, bestehen demnach in einer Blutdrucksenkung, Verlangsamung der Atmung und in einer sehr ausgesprochenen Wirkung auf das Zentralnervensystem. Die letztere Wirkung, welche schon von

1) Versuche im Kaiser Wilhelm-Institut (vgl. S. 7). 
Weber ${ }^{1}$ ) ausführlich beschrieben wurde, besteht in heftigen konvulsivischen Krämpfen, die anfallsweise auftreten, im Verlaufe der Vergiftung an Heftigkeit zunehmen, dann allmühlich wieder schwächer werden, um schliesslich in Koma, Lähmung und Tod überzugehen. Alle diese Wirkungen kommen am deutlichsten zur Entwicklung bei Vergiftungen nach intravenöser Injektion und mit grossen Dosen.

Bei intravenöser Injektion sehr geringer Dosen zeigt das Krankheitsbild einen wesentlich anderen Verlauf. Es entwickelt sich lediglich in schleichendem Verlaufe unter allmählicher Gewichtsabnahme eine toxische Kachexie, welche im Verlaufe von Tagen oder Wochen zum Tode führt. Im Versuch 5 genügten hierzu im ganzen $0,075 \mathrm{~g}$ käufliches Dimethylsulfat, welche nach der Analyse $0,051 \mathrm{~g}$ reinem Dimethylsulfat entsprechen. Dieses Resultat steht vollständig im Einklang mit den Erfahrungen, welche an Katzen nach Einatmung von Dimethylsulfat gemacht worden sind. Hier ergab sich noch eine tödliche Wirkung nach Einatmung einer Menge von 500 und in einzelnen Fällen selbst 250 ct, wenn 0 die Konzentration des Gases ausdrückt in Kubikmillimetern der flüssigen Substanz pro Kubikmeter Luft und $t$ die Versuchsdauer in Minuten bedeutet. Unter der Annahme eines Minutenvolumens der Atmung von 30 Litern für eine ausgewachscne Katze berechnet sind aus diesen Zahlen als kleinste tödliche Mengen etwa $8-16 \mathrm{mg}$. Dieses auffallende Ergebnis, wonach das Dimethylsulfat, ähnlich wie eine Reihe anderer als Gaskampfstoffe verwendeter Substanzen, die stärksten „traditionellen Gifte" wie etwa den Zyanwasserstoff um ein vielfaches übertrifft an Wirksamkeit, ist in vielfachen Versuchen bestätigt gefunden worden. Allerdings benötigt man dazu die Anwendung der im Kaiser Wilhelm-Institut für diese Versuche ausgearbeiteten Methodik ${ }^{2}$ ). Das Krankheitsbild entwickelt sich in diesen Fällen genau wie in dem obigen Versuch 5 und kann auch nach der Einatmung derartiger geringer Dosen nach drei bis vier Wochen zum Tode führen, ohne dass mehr als ganz leichte und nur vorübergehende Reizerscheinungen von Seiten der Atmungsorgane aufgetreten wären. Im einzelnen vergleiche man hierzu das weiter unten als dritten Krankheitstypus beschriebene Vergiftungsbild.

1) 1. c.

2) Umfangreiche Versuche im Kaiser Wilbelm-Institut baben orgeben, dass dio allgomein üblichen Glasglockenversuche nicht geeignet sind die kleinsten wirksamen Dosen giftiger Gase zu ermitteln, weil ihre Oberfäche im Vergleich zum Volumen zu gross ist, so dass durch die Adsorption der Gase an der Oberfläche der Versuchsglocken Fehler von vielen hundert Prozent entsteben. Um diese Febler zu vermeiden, war es notwendig, Versuchsräume von mindestens $1 \mathrm{cbm}$ Inhalt für Mäuse und einzelne Katzen, und noch grössere Versuchsräume von 10 und $40 \mathrm{cbm}$ Inhalt für mehrere und grössere Versuchstiere und Menschen zu konstruieren. Auch in diesen grossen Versuchsräumen spielt die Adsorption der Gase an den Wandungen noch oine erbebliche Rolle. W'io zahlreiche Analysen gezeigt haben, muss im Durchschnitt bei einer Versuchsdaver von höchstens 20 Minuten mit einem Konzentrationsabfall von $50 \mathrm{pCt}$. gerechnet werden. Um auch diese Ungenauigkeit zu vermeiden, wurde ein derartiger Versuchsraum mit einer Strömungsapparatur erbaut. Eine genauere Beschreibung dieser Einrichtungen, welche, um gut zu funktionieren, sehr vieler technischer Hilfsmittel bedürfen, kana hier naturgemäss nicht Platz finden. 
II.

Unter Berücksichtigung der Versuche ergibt sich also folgende Auffassung von dem Mechanismus der Wirkung des Dimethylsulfats. Seine gesamte Wirkung wird hervorgerufen:

1. durch das unzersetzte Molekül und

2. durch die im Organismus entstehenden Spaltprodukte, in erster Linie also durch das Säureradikal ${ }^{1}$ ).

Dabei hat man sich vorzustellen, dass der Ester, welcher durch die Blutbahn in den Organismus eingeführt wird, dort alsbald gespalten wird, dass aber die abgespaltene Säure im Blute keine wesentlichen Veränderungen hervorzurufon vermag, weil das Blut vermöge seines Alkaligehaltes und wegen der relativen Unempfindlichkeit des Serumeiwcisses durch die in betracht kommenden Säuremengen noch nicht wesentlich alteriert wird. Gelangt dor Ester jedoch ohne Vermittlung des Blutes direkt mit den Organen in Berührung, so tritt die Säurewirkung viel stärker in die Erscheinung, weil erstens die Neutralisation durch den Alkaligehalt des Blutes wegfällt und weil andererseits das Organeiweiss in weit höherem Masse gegen Säure empfindlich ist als das Serumeiwciss. Gegen die Auffassung von Weber ${ }^{2}$ ), dass die Bedeutung des Alkyls wegen seiner geringen Quantität für die Wirkung belanglos sei, spricht wohl der grosse Einfluss, den die Alkylgruppe auf die Giftigkeit der Substanzen besitzt, dass also z. B. Dimethylsulfat giftiger ist als das Diäthylsulfat. Ihre Lirklärung im Vergleich zu der Giftigkeit der freien Alkohole findet diese Tatsache durch die Annahme, dass die $\Lambda$ bspaltung erst am Orte der Wirksamkeit vor sich geht. Ein Argument für dic Zulässigkeit dieser Auffassung ist z. B., dass Chlordimethylsulfat ${ }^{3}$ ), welches sich mit kaltem Wasser langsam in Methylschwefelsäure, Formaldehyd und Chlorwasserstoff zersetzt, wesentlieh giftiger ist als jedes der genannten Zersetzungsprodukte für sich.

Die entwickelte Auffassung lässt sich dahin verallgemeinern, dass man in dem Gesamtbilde einer durch irgend einen Ester horvorgerufenen Wirkung nebeneinander die Wirkung des Gesamtmolcküls und der Spaltprodukte anzunehmen hat, und ist insbesondere von Interesse für dic Erklärung der Wirkung zahlreicher Gaskampfstoffe, welche häufig auf eine reine Säurewirkung zurückgeführt worden ist. Diese Auffassung mag berechtigt sein bei Substanzen wie dem Phosgen oder dem Perchlorameisensäuremethylester, welche mit Wasser momentan unter lebhafter

1) Nach Fertigstellung der Arbeit wurde mir bekannt, dass Pernon Lynch, HomerW.Smith und E.K.Marshall gelegentlich einer Untersuchung über Dichloräthylsulfid auch durch einige Beobachtungen über die Wirkung des Dimethylsulfat ebenfalls zu der Auffassung gelangen, dass diese Wirkung auf intrazellulärer Säureabspaltung beruht. - Chem. Zentralbl. 1919. IIl. 28.

2) 1. .. S. 124 .

3) Nach Versuchen im Kaiser Wilhelm-Institut liegt die tödliche Dosis

$$
\begin{aligned}
& \text { für Chlordimethylsulfat etwa bei } \quad 1500 \text { et } \\
& \text { "Formaldehyd " " } 6000 \text { " } \\
& \text { "Chlorwasserstoff " " über } 30000 "
\end{aligned}
$$


Gasentwicklung zersetzt werden. Im Gegensatz dazu darf man bei Verbindungen, welche sich sehwerer mit Wasser zersetzen, wie etwa Chlorpikrin unter geeigneten Vergiftungsbedingungen das Auftreten resorptiver Erscheinungen erwarten, zumal da sowohl die Befunde an Tieren hierfür sprechen (zentrale Lähmungserscheinungen nach grösseren Dosen) wic auch einzelne Fälle am Menschen. Dem widerspricht nicht, wenn beim Menschen bei den im Felde entstandenen Gasvergiftungen resorptive Erscheinungen tatsächlich nur selten aufgetreten sind, da die Bedingungen, unter denen die Vergiftung stattfand, namentlich die sehr niedrigen Konzentrationen, für das Zustandekommen resorptiver Wirkungen im allgemoinen nicht günstig waren.

Für den quantitativen Grad der Giftigkeit sind in hohem Masse dic physikalischen Eigenschaften der einzelnen Ester verantwortlich zu machen, in erster Linie die Wasserlöslichkeit, sodann die Zersetzlichkeit durch Wasser, die Lipoidlöslichkeit usw. So wird beispielsweise Dimethylsulfat annähernd doppelt so schnell rom Wasser zersetzt wie Diäthylsulfat. Daraus wird verständlich, dass die Aetzwirkung des Dimethylsulfats stärker ist als die des Diäthylsulfats. Unter Umständen ist viclleicht auch die Wirkung auf die Körperfermente in betracht zu ziehen, da sich gezeigt hat, dass Dimethylsulfat die Wirkung der Blutkatalase noch in einer Verdünnung von 1:50000 deutlich hemmt ${ }^{1}$ ).

Der angenommene Mechanismus dor Giftwirkung erklärt auch die Tatsache, dass die verschiedene Applikationsweise von ciner gewissen Bedeutung für die Art ist, in der das Gift seine Wirkung ausübt. Sie ist von Bolang für dic Zeitdauer, welche von Beginn der Vergiltung bis zum Eintritt der ersten Wirkung verstreicht (die Latenz- oder Inkubationszeit $^{2}$ ), sodann dafür, ein wie grosser Bruchteil des eingeführten Giftes an den Ort der spezifischen Wirkung gelangt. Infolgedessen beeinflusst die $\Lambda$ rt der $\Lambda$ pplikation sowohl den Charakter des Krankheitsverlaufes als auch die Schwere der Erkrankung.

Insbesondere ist es mit Beziehung auf dic Gaskampfstoffe von Interesse, den Unterschied zwischen intravenöser Injektion und Einatmung durch die Jungen zu erörtern. Bei ciner Substanz mit einer ganz exquisiten spezifischen $\Lambda$ ffinität zum Nervengewebe, nämlich beim Strychnin ${ }^{3}$ ) liess sich zeigen, dass die wirksamen und letalen Dosen bei der Aufnahme durch die Lungen dio gleichen sind wie bei der intravenösen Injektion, dass also prinzipiell intravenöse Injektion und Einatmung gleichwertig sind, wobei jedoch zu bedenken ist, dass bei der Einatmung durch die Lungen die Vereinigung des Giftes mit dem Blute sich auf einer vicl grösseren Oberfläche vollzicht und daher unmittelbar eine sehr verdünnte Lösung und infolgedessen cine gleichmässigere Verteilung im Organismus zu stande kommt. Man kann sich eine Vorstellung davon machen, wenn man berücksichtigt, dass die Oberfläche der menschlichen Lunge

1) Versuche des Verf. im Kaiser Wilhelm-Institut.

2) Die Latenzzeit ist für die militärische Beurteilung von Gasen von Bedeutung, da von ihrer Dauer die taktische Verwendung der einzelnen Kampfstoffe abhängt.

3) Versuche im Kaiser Wilbelm-Institut von Herm Dr. Th. A. Maas. 
auf $80 \mathrm{qm}$ anzunehmen ist. Hiernach ergibt sich, dass je nach den physikalisehen Eigenschaften der Stoffe das Krankheitsbild bei verschiedener Applikation ganz verschicden verläuft, indem die einzelnen Sypmtome in ganz anderer Reihenfolge auftreten bzw. überhaupt nicht erscheinen, wenn z. B. bei der Einatmung die Dosis nicht gross genug ist, um neben der lokalen Wirkung noch einen Teil zur Resorption gelangen zu lassen. Die allgemeine Pathologie derartiger Vergiftungen lässt sich daher nur getrennt darstellen. Der klinische Verlauf ist ganz verschieden, je nachdem die funktionellen Wirkungen oder die anatomischen Veränderungen das Bild beherrschen.

Zum Vergleiche wurde zuerst das auch sehon von Weber ${ }^{1}$ ) berücksichtigte Diäthylsulfat herangezogen, dessen Wirkung bei intravenöser Injektion derjenigen des Dimethylsulfats durchaus analog ist, wie aus den folgenden Versuchen hervorgeht.

In chemischer Beziehung ist das Diäthylsulfat dem Dimethylsulfat weitgebend ähnlich. Es ist eine schwere, in Wasser schwer lösliche, pfefferminzähnlich riechende Flüssigkeit, die bei $208^{\circ}$ fast unzersetzt siedet. In Wasser sinkt es unter, beim starken Schütteln mit der 20 fachen Menge Wassers löst sich ein grosser Teil, während der Rest mehr oder weniger fein emulgiert wird. Die frisch bereiteto Lösung von $0,5 \mathrm{ccm}$ Diäthylsulfat in $40 \mathrm{ccm}$ Wasser wird bei Zusatz von Phenolphthalein durch einen Tropfen $\mathrm{n} / 10$ Natronlauge gerötet. Nach kurzer Zeit tritt jedoch Entfärbung ein. Zum Neutralisieren waren erforderlich:

nach 1 Stunde..... 1,7 ccm n/10 NaOH

nach 25 Stunden ... 18,0 ccm n/10 NaOH

also im Ganzen ..... 19,7 ccm n/10 NaOH

entsprechend $0,0946 \mathrm{~g}$ Schwcfelsäure und 0,1456 g Diäthylsulfat. Diäthylsulfat wird also von Wasser wesentlich langsamer hydrolysiert als Dimethylsulfat. Hierdurch wird verständlich, dass die Aetzwirkung des Diäthylsulfats wesentlich geringer ist, als die des Dimethylsulfats. Nach den Versuchen im Kaiser Wilhelm-Institut ist das Diäthylsulfat bei der Einatmung etwa $10 \mathrm{Mal}$ weniger giftig als das Dimethylsulfat. Die niedrigste tödliche Dosis für die Katze betrug 3000 ct.

Ueber die Wirkung des Diäthylsulfats bei intravenöser Injektion orientiert der folgende

8. Versuch: Kaninchen $1300 \mathrm{~g}, 0,5 \mathrm{ccm}$ Diäthylsulfat mit $10 \mathrm{ccm}$ physiologischer Kochsalzlösung unter Zusatz von einem 'Tropfen Phenolphthalein mit Natronlaug $\theta$ leicht alkalisch gemacht werden durch starkes Schütteln emulgiert. Von dieser Emulsion werden 5 ccm in die rechte Halsvene injiziert. Nach 2 Minuten verschwinden sämtliche Reflexe, Auftreten leichter Krämpfe, Trismus und Atmungsverlangsamung. Nach 3 Minuten Injektion von weiteren $3 \frac{1}{2} \mathrm{ccm}$ der Lösung. Während der Injelition tritt eine geringe Blutdruckschwankung ein, ausserdem Atnungsverlangsamung nach 7 Minuten starke Blutdrucksenkung, das Kaninchen schreit, nach 9 Minuten vollständige Blutdrucksenkung, Respirationshemmung und Exitus. Die Sektion ergibt keine bemerkenswerten anatomischen Veränderungen.

In diescm Versuche haben $0,425 \mathrm{~g}$ Diäthylsulfat die tödliche Wirkung erzielt. Die Substanz ist also bei intravenöser Anwendung annähernd

1) 1. c. p. 124 . 
von gleicher Giftigkeit wie das Dimethylsulfat, was im Vergleich zu der geringen Aetzwirkung bemerkenswert ist. Die Wirkungen auf das Zentralnervensystem sind bei beiden Stoffen die gleichen $\mathrm{Da}$ sich nun das Diäthylsulfat schwerer zersetzt als das Dimethylsulfat, so spricht das Ausbleiben der Actzwirkung bei dem ersteren dafür, dass die funktionellen und zentralen Wirkungen durch das Gesamtmolekül, die Aetzwirkung dagegen durch die Säureabspaltung bewirkt wird.

Den letzteren Schluss vermochte Weber nicht zu ziehen, weil er von der irrigen Voraussetzung ausgeht, dass sich "das Diäthylsulfat noch leichter zersetzt als der Dimethylester $\left.{ }^{1}\right)^{\complement}$.

Um nun auch den Einfluss des Säureradikals auf den Charakter der Wirkung zu ermitteln, wurde eine Anzahl weiterer Versuche angestellt, von denen hier zunächst die Ester der Phosphorsäure und der Kohlensäure angeführt werden sollen.

\section{Phosphorsäuremethylester.}

9. Versuch. Kaninchen, $1700 \mathrm{~g}$, erbält von der 5 proz. mit Natronlauge neutralisierten Lösung $3 \mathrm{ccm}$ in die rechte Halsvene injiziert. Nach einer wenige Sekunden dauernden Blutdruckerböhung folgt өine geringe Blutdrucksenkung und veränderter Puls; nach 26 Minuten werden weitere $3 \mathrm{ccm}$ injiziert. Erfolg: Atmungsverlangsamung und Blutdruckschwankungen, welche innerhalb von weiteren 6 Minuten zum Exitus unter Blutdrucksenkung und Atemstillstand führen. Die Sektion ergibt keine anatomischen Veränderungen von Belang.

Die Kurve zeigt also den gleichen Charakter wie nach Dimethylund Diäthylsulfat.

\section{Kohlensäuredimethylester ${ }^{2}$ ).}

Kohlensäuredimethylester ist eine ätherisch riechende, in Wasser und allen organischen Lösungsmitteln leicht lösliche Flüssigkeit, welche bei $91^{\circ}$ siedet.

10. Versuch. $9 \mathrm{ccm}$ einer 5 proz. Lösung, welche einem Kaninchen von $1940 \mathrm{~g}$ in die rechte Halsvene injiziert wurden, verursachten keine Veränderung von Blutdruck und Atmung.

11. Versuch. Lässt man ein Kaninchen Dimethylkarbonat einatmen, so folgt auf ein kurzes Exzitationsstadium eine leichte Narkose mit vertiefter Atmung und erhaltenen Kornealreflexen. Nach 4-5 Minuten ist die Narkose wieder aufgehoben, das Kaninchen schwankt noch etwas, nach 7-8 Minuten völlige Wiederherstellung, in den nächsten 24 Stundeu keine krankbaften Erscheinungen, im Urin kein Eiweiss undZucker.

12. Versuch. Setzt man ein Meerschweinchen oder eine Ratto den Dämpfen von Dimethylkarbonat in einer Konzentration von $0,4 \mathrm{~g}$ pro Liter unter einer Glasglocke aus, so zeigen die Versuchstiere nach einer Minute Unruhe, Nasenreiz, Reizung der Bindehäute, Augentränen; die Reize verschwinden nach 3 Minuten langer Einwirkung, eine narkotische Wirkung tritt jedoch bei diesen Konzentrationen noch nicht ein.

Das Dimcthylkarbonat verursacht also bei der Einatmung und bei der intravenösen Injektion eine sehr oberflächliche und flüchtige Narkose,

1) 1. c. S. 125 .

2) Von den Chemischon Werken Grenzach in dankenswerter Weise zur Verfügung gestellt. 
ohne in den angewandten Dosen eine Veränderung von Blutdruck und A tmung zu bewirken. Wegen der Reizwirkung auf die Schleimhäute wie auch wegen der geringen Wirksamkeit kommt es als praktisches Narkotikum nicht in Betracht.

Mit den vorstehenden korrespondiert ein Versuch mit dem

Kohlensäurediäthylester ${ }^{1}$ ).

Diäthylkarbonat ist eine ätherähnlich riechende, in organischen loösungsmitteln leicht, in Wasser schwer lösliche Flüssigkeit vom Sicdepunkt $126^{\circ}$.

13. Versuch. $0,5 \mathrm{ccm}$ des Präparates werden mit $20 \mathrm{ccm}$ physiologischer Kochsalzlösung zu ciner leidlich haltbaren Emulsion gemischt. $10 \mathrm{ccm}$ yon dioser Emulsion einem Kaninchen von $1300 \mathrm{~g}$ in die Vena jugularis injiziert verursachen eine tiefo Narkose unter Verschwinden der Reflexe, wolche narh Verlauf von 5 Minuten unter Rückkehr der Reflexe wiedor gelöst wird. Während der ganzen Dauer des Versuchs wurde keine Beeinflussung von Blutdruck und Almung beobachtet.

Um nun als Säureradikal eine hochgiftjge organische Säure zu verwonden, wurde der

\section{Oxalsäureäthylester}

unter Bcobachtung von $\Lambda$ tmung und Blutdruck untersuchi.

Oxalsäureäthylester ist eine ätherähnlich riechende Elüssigkeit vom Sicdepunkt $186^{\circ}$, in Wasser löslich.

14. Versuch. Kaninchen, $2200 \mathrm{~g}$, erhält von einer 5 proz. mit Soda noutralisierten Jösung zweimal je $5 \mathrm{ccm}$ in die Vena jugularis injiziert. Erfolg: Geringe Blutdrucksenkung. Nach 12 und 17 Minuten werden woitere je $5 \mathrm{ccm}$, nach 18 Minuten $31 / 2 \mathrm{ccm}$, nach 32 Minuten zuerst $3 \mathrm{ccm}$, wenige Sekunden später noch $2 \mathrm{ccm}$ injiziert, und rufen 2wei flüchtige Blutdrucksenkungen mit $\Lambda$ tmungsspindeln hervor. Erst weitere $4 \mathrm{ccm}$ nach 37 Minuten bewirken tiefe Blutdrucksenkung mit Atmungsspindel, daran anschliessend eine kurze Erhöhung des Blıtdruckes mit alsbald wieder beginnender Blutdrucksenkung und Respirationshemmung, welche innerhalb von 3 Minuten zum Tode fübren.

Dio vorstchend beschriebene Kurve könnte so gedeutet werden, dass dic flüchtige Blutdrucksenkung, welche von der spindelförmigen Atmungskurve begleitet wird und nach wonigen Sekunden zur Norm zurückkehrt, als Wirkung des unzersetzten Esters aufzufassen ist, während die endgültige, zum Exitus führende, irreversible Senkung auf eine Wirkung der abgespaltenen Oxalsäure zurückgeführt wird. Die injizierte Dosis von $1,6 \mathrm{~g}$ Oxalsäuroäthylester entspricht etwa $1,0 \mathrm{~g}$ Oxalsäure.

Es folgen nun noch eine Reihe weiterer neutraler Ester, wclche in der Art ihrer Wirkung den vorstehend beschriebenen gleiehen.

A meisensäurcäthylester.

$\Lambda$ meisensüureäthylester ist eine angenchm riechende Flüssigkeit vom Siedepunkt $54,5^{\circ}$, welche in Wasser leicht löslich ist und ausserordentlich schnell hydrolysiert wird. 8,5 bzw. $10 \mathrm{cem}$ der 5 proz. neutralisierten

1) Von den Chemischen Werken Grenzach zur Verfügung gestellt. Die anderen Verbindungen sind die im Handel käulichen Produkte (Kahlbaum \& Schuchardt). 
Lösung haben überhaupt keine sichtbare Wirkung, vermutlich, weil sie nichts anderes enthalten als ameisensaures Natrium und Aethylalkohol.

Ameisensäurepropylester,

welcher eine ätherisch riechende Flüssigkeit vom Siedepunkt darstellt, ruft nach Injektion von $8,5 \mathrm{ccm}$ der 5 proz. Lösung cbenfalls keinerlei Wirkung hervor.

\section{Essigsäureäthylester.}

Essigsäureäthylester ist eine angenehm riechende, in Alkohol, Aether, Chloroform und auch in Wasser lösliche Flüssigkeit vom spezifischen Gewicht 0,92 und rom Siedepunkt $77^{\circ}$.

15. Versuch. $9,5 \mathrm{ccm}$ der 5 proz. Lösung, intravenös injiziert, verursaeben keinerlei Aenderung von Blutdruck und Atmung.

$$
\text { Essigsäurepropylester. }
$$

16. Versueh. Essigsäurepropylester vom Siedepunkt $105^{\circ}$ in 5 proz. neutralisierter Lösung verursacht bei intravenöser Injektion von $5 \mathrm{ccm}$ der Lösung keine Wirknng. Nach 9 Minuten weitere 4,5 ocm injiziert bewirken eine sehr flüchtige Blutdrucksenkung mit Respirationshemmung, tiefer Narkose und sehr schwachen Reflexen, nach einer Minute völlige Rückkehr zur Norm.

\section{Bromessigsäureäthylester.}

Bromessigsäuräthylester ist eine schwor bewegliche Flüssigkeit vom Siedepunkt $159^{\circ}$ und stechendem Geruch, äusserster Reizwirkung auf sämtliche Schleimhäute, löslich in organischen Lösungsmitteln, in Wasser unter Gasentwicklung.

Wegen seiner starken Reizwirkung ist der Bromessigsäureäthylester im Anfang des Krieges als Gaskampfstoff verwendet worden, und wurde aus diesem Grunde auch im Kaiser Wilhelm-Institut untersucht. Die tödliche Dosis war nach den dortigen Versuchen kleiner als 5000 ct bei der Einatmung durch die Lungen. Bei subkutaner Anwendung an Katzen bewirkten

$$
\begin{aligned}
& 1,0 \mathrm{ccm} \text { den Tod nach } 15 \text { Minuten } \\
& 0,2 \quad " \quad " \quad 1 \text { Stunde } \\
& 0,1 " n n \pi 24 \text { Stunden. }
\end{aligned}
$$

Dic bei diesen Vergiftungen zu Tage tretenden Erscheinungen von seiten des Zentralnervensystems bestanden in Ataxie und Lähmungen.

Ueber die Wirkung bei intravenöser Anwendung orientiert der folgende

17. Versuch. Von einer 5 proz. Lösung, welche nach Beendigung der Gasentwicklung schwach alkalisch gemacht worden ist, wird 1,0 ccm einem Kaninchen von $1940 \mathrm{~g}$ in die Vena jugularis injiziert. Nach 2 Minuten tritt unter Blutdrucksenkung Tod durch Respirationslähmung ein.

Der Versuch ist insofern nicht unbedingt überzeugend, als die angewendete Lösung nicht den unveränderten Bromessigester, sondern nur sein Zersetzungsprodukt mit Wasser enthielt. Jedoch würde bei der Geschwindigkeit, mit der diese Zersetzung vor sich geht, auch in vivo für resorptive Wirkungen dieses Zersetzungsprodukt in Betracht kommen, so dass der Versuch also trotzdem berechtigt ist. Die tödliche Dosis würde nach diesem Versuche weniger als $50 \mathrm{mg}$ betragen, also weniger als $25 \mathrm{mg}$ pro Kilogramm Körpergewicht Kaninchen. 
Das anatomische Krankheitsbild, welches von dem Bromessigester erzeugt wird, entspricht dem weiter unten beschriebenen ersten Krankheitstypus. Die am Menschen beobachteten Vergiftungen entsprechen den Beobachtungen an Tieren ${ }^{1}$ ).

Als neutrale Ester einer zweibasischen organischen Säure wurden schliesslich noch die Ester der Malonsäure untersucht. Hierüber folgende Versuche:

Malonsäuredimethylester.

Malonsäuredimethylester ist eine ätherähnlich riechende, bei $181^{\circ}$ siedende Flüssigkeit, welche in organischen Lösungsmitteln leicht löslich ist und mit Wasser emulgiert werden kann.

18. Versuch. Von einer 5 proz. Emulsion in physiologischer Kochsalzlösung werden einem Kaninchen von $1940 \mathrm{~g} 9 \mathrm{ccm}$ in die Halsvene injiziert, welche im Verlaufe von 12 Minuten keinen Einfluss auf Blutdruck und Atmung ausüben. Die Reflexe sind während des Versuches dauernd erhalten.

\section{Malonsäurediäthylester.}

Malonsäurediäthylester ist eine ätherisch riechende Flüssigkeit vom spezifischen Gewicht 1,068, welche bei $198^{\circ}$ siedet. Verhalten zu Lösungsmitteln wie bei dem Methylester.

19. Versuch. $5 \mathrm{ccm}$ einer Emulsion in physiologischer Kochsalzlösung einem Kaninchen von $1400 \mathrm{~g}$ intravenös injiziert verursachen alsbald eine in wenigen Sekunden vorübergehende Narkose mit Blutdrucksenkung und spindelförmiger Atmungskurve. Nach 5 Minuten werden weitere $3 \mathrm{ccm}$ der Emulsion injiziert. Erfolg: Tiefo Narkose unter Aufhebung der Reflexe, starke Blutdrucksenkung, Verflachung und Verlangsamung der Atmung, nach 2 Minuten völlige Rückkehr zur Norm.

Um schliesslich auch noch den Ester einer aromatischen Säure zu prüfen, wurden in dem folgenden

20. Versuch dem Kaninchen $2200 \mathrm{~g}$ Benzoesäuremethylester in 5 proz. neutraler Lösung intrayenös injiziert. $9,5 \mathrm{ccm}$ hatten keinerlei Wirkung.

Die Versuche bestätigen also die Richtigkeit der oben dargestellten Auffassung von der Giftwirkung des Dimethylsulfats.

Die funktionellen Veränderungen, welche die untersuchten Ester hervorrufen, bestehen demnach stets in einer Blutdrucksenkung, mit der in ausgesprochenen Fällen eine Verlangsamung und Verflachung der Atmung einhergeht. Daneben wirken die Ester auch auf das Zentralnervensystem, indem ein Teil von ihnen, wie die Ester der Mineralsäuren, Krämpfe verursachen, ein anderer Teil, wie die Ester der Kohlensäure und der Fettsäuren, eine flüchtige narkotische Wirkung haben.

$$
\text { III. }{ }^{2} \text { ) }
$$

Zur Vervollständigung der Darstellung soll noch ein kurze Uebersicht über die anatomischen Erscheinungen gegeben werden, welche nach der Einatmung von Dimethylsulfat auftreten, zumal da diese Erschei-

1) Nach Versuchen im Kaiser Wilhelm-Institut.

2) Dieser Abschnitt ist ein kurzes Referat nach einem ausführlichen Bericht über die Beobachtungen zur Pathologie der Kampfgasvergiftung auf Grund der Tier- 
nungen ausser beim Dimethylsulfat bei mehreren hundert anderen Stoffen, welche als Gaskampfstoffe für die Kriegsmedizin und als gewerbliche Gifte für die Fabrikhygiene von Interesse sind, auftreten.

Die durch das Dimethylsulfat bei der Einatmung hervorgerufenen Erkrankungen sind je nach den Versuchsbedingungen, unter denen die Vergiftung entstand, verschieden. Konzentration, Einatmungszeit, Temperatur, Luftfeuchtigkeit und vielleicht noch viele andere Umstände können bei der gleichen absoluten Menge des eingeatmeten Giftes verschiedene der nachstehend zu beschreibenden Krankheitstypen verursachen.

Vorweg sei bemerkt, dass die beobachteten Erkrankungsformen sich als Misch- oder Uebergangsformen auffassen lassen zwischen drei wesentlich von einander verschiedenen, ausgeprägten Krankheitstypen. Diese drei Krankheitstypen selbst kamen ebenfalls zur Beobachtung, jedoch verhältnismässig viel seltener, als die Uebergangsformen. Sie stellen daher gewissermaassen Idealformen dar.

Die erste typische Erkrankungsform besteht in einem Oedem der Lunge, welches durch die zerstörende Einwirkung des Dimethylsulfats auf die Epithelien der Lungenalveolen und die Lungenkapillaren hervorgerufen wird. Das eingeatmete Dimethylsulfat bewirkt eine Schädigung der Alveolarepithelien, wodurch das Eindringen einer plasmaähnlichen Flüssigkeit aus dem Blut in die Lungenalveolen ermöglicht wird. Infolgedessen wird die atmende Oberfläche in gefährlichem Masse verkleinert, das Blut wird eingedickt, der Kreislauf verlangsamt, für das Herz entsteht infolge der vermehrten Viskosität des Blutes eine erhöhte Arbeit, daneben Sauerstoffmangel und erschwerte Kohlensüureabfuhr, welche eine Erstickung des Herzens herbeiführen.

Diesen Verhältnissen entsprechend zeigt das anatomiscche Bild ein hochgradig entwickeltes $\mathrm{J}$ ungenödem, die Lunge kann auf das fünfbis siebenfache ihres normalen Volumens und Gewichts vergrössert sein; das Herz ist prall mit dunklem, fast schwarzem Blut gefüllt, welches im linken Ventrikel meist flüssig ist, in der rechten Herzhälfte dicke schwarze Kruorgerinnsel, im rechten Vorhof manchmal - bei längerer Krankheitsdauer — auch Speckhautgerinnsel enthält. Die Nierenrinde ist hellgrau bis gelbgrau, das Mark hellviolett bis dunkelrot, die Markrindengrenze sehr viel dunkler gefärbt. Die Harnkanälchen sind erweitert, im Interstitium treten punktförmige Blutungen auf, das Epithel zeigt Verfettungen und Degenerationen, Bildung von Zylindern. In diesem Zustande scheidet die Niere Eiweiss aus. In allen Organen treten Stauungserscheinungen auf.

Der zweite Krankheitstypus tritt beim Dimethylsulfat ebenfalls in sehr ausgeprägter Form auf. Er besteht in einer pseudomembranösen (fibrinösen, kruppösen) Pharyngitis, Laryngitis, Tracheitis und Bronchitis. Daneben besteht kein Oedem der Lunge.

versuche im Kaiser Wilhelm-Institut, welchen Verfasser im Juni 1917 der Medizinalabteilung des Kgl. Preuss. Kriegsministeriums vorgelegt hat. Vgl. hierzu auch Robert Müller, Zeitschr. f. exp. Path. u.Ther. 1901. Bd. 9. S. 103. 
Auch bei dieser Erkrankungsform handelt es sich um Schädigung des Epithels, das nekrotisiert und teilweise abgestossen wird. Das überflutende Exsudat gerinnt und bildet Pseudomembranen, welche sich sehr leicht abheben lassen. Das Lungengewebe selbst zeigt dabei nur geringfügige Veränderungen wie vereinzelte Blutungen, kleinere Infarkte oder dergleichen. Vor allen Dingen tritt kein primäres Oedem der Lunge auf. Das Iumen der Luftwege kann bei diesem Krankheitsbilde so mit Pseudomembranen ausgefüllt werden, dass os schliesslich nur noch für eine Stricknadel durchgängig ist. Der Tod wird in diesen Fällen durch mechanische Erstickung hervorgerufen.

Weiterhin findet sich bei diesem Krankheitstypus Glottisödem, Entzündung der regionären Lymphdrüsen des Halses und der Lungen, Schwellung und Hyperämie der Milz. Eine Schädigung der Nieren kann unter Umstäuden vorhanden sein.

Bei den bisher beschriebenen beiden typischen Krankheitsbildern steht die ätzende Wirkung des Dimethylsulfates auf die bei seinem Eintritt in den Organismus zunächst gelegenen Organe im Vordergrunde. Die folgenden Erkrankungsformen sind dadurch charakterisiert, dass die gesamten Atmungsorgane keine oder nur ganz unbedeutende Veränderungen aufweisen. In diesen Fällen wird das Gift also resorbiert und zeigt seine Wirkung mehr oder wenigor ausgesprochen an einzelnen oder gleichzeitig an mehreren lebenswichtigen Organen, insbesondere an Herz, Niere und Nebenniere, Leber, sowie gelegentlich an den Speicheldrüsen und schliesslich auch am Nervensystem.

Die resorptive Form der Vergiftung führt demnacb eine allgemeine Gewebsschädigung herbei, welche wenigstens teilweise auch als eine spezifische Schädigung der Kapillargefässe aufgefasst werden muss. Meistens steht hier im Vordergrunde eine schwere Erkrankung des Herzmuskels, die zum Ausdruck kommt in einem schlaffen, dilatierten Herzen, mikroskopisch in einer Degeneration der Muskelfasern und punktförmigen Blutungen im Herzmuskel, Epi- und Endokard. In den Nieren von Katzen, Hunden fanden sich gelegentlich alle Grade von Epithelschädigung, daneben Blutungen, Oedem. Besonders auffallend ist, dass nicht allzu selten in den Nebennieren Blutungen unter der Kapsel und im Parenchym auftreten. Schwere Erkrankungen des Norvensystems bedeuten die in der Literatur bereits beschriebenen, nach grossen Dosen auftretenden Erscheinungen von Koma, Krämpfen und Konvulsionen.

Insbesondere die ersten beiden Formen der Erkrankung nach Dimethylsulfat sind überaus charakteristisch. Bei weitem häufiger jedoch begegnet man den Mischformen, deren Einzelerscheinungen im Hinblick auf die beschriobenen Idealformen gedeutet werden können, so dass hiernach eine Analyse der Sektionsbefunde im besonderen Falle ganz leicht durchzuführen ist.

Hieraus ergibt sich zunächst, dass der Verlauf der einzelnen Vergiftungen sehr verschieden sein kann. Von ganz akut verlaufenden Fällen, welche unter den schwersen Erscheinungen von seiten der Atmungsorgane oder des Zentralnervensystems innerhalb weniger Stunden zum Tode führen, bis zu solchen, die unter den Erscheinungen einer 
Herzmuskelschwäche oder unter dem Bilde einer schweren toxischen Kachexie nach wochenlangem schleichenden Verlauf zum Tode oder zu Genesung führen, finden sich alle Uebergänge.

Um die Bedingungen zu ermitteln, unter welchen das eine oder das andere Krankheitsbild aufzutreten pflegt, würde man einer überaus grossen Zahl von Versuchstieren bedürfen. Deshalb können hierüber nur einige Vermutungen aufgestellt werden, insoweit das bereits vorliegende Material zu solchen zu berechtigen scheint. Zunächst darf wohl mit einiger Sicherheit angenommen werden, dass sehr hohe Dosen gleichgültig, auf welchem Wege sie in den Organismus eingeführt werden, mindestens zu einer teilweisen Resorption in unveränderter Form und demgemäss $z u$ Erscheinungen von seiten des Nervensystems und des Herzens führen. Ferner scheinen längere Zeit hindurch in niedrigster Konzentration aufgenommene Dosen, insbesondere per pulmones, ohne wesentliche lokale Reizerscheinungen resorbiert zu werden und dann die schleichend verlaufenden Formen mit Herzschwäche und Kachexie zu entwickeln. Dabei ist anscheinend die absolute Dosis weniger massgebend als die Konzentration und Zeitdauer, während deren das Gift einzuwirken Gelegenheit hat. Inwieweit noch klimatische Einflüsse, insbesondere auch dic Prädisposition der verschiedenen Tierarten oder gar Individuen eine Bedeutung für das Auftreten der verschiedenen Erkrankungsformen haben, ist natürlich ohne Zugrundelegung eines grossen Vorsuchsmaterials für diesen besonderen Zweck zu erörtern unmöglich. Immerhin wäre die Klarstellung dieser Verhältnisse von grosser allgemeiner Bedeutung, da sie nicht nur für das Dimethylsulfat, sondern wie die gesamten vorstehenden Ausführungen auch für sämtliche Gaskampfstoffe und gewerbehygienisch wichtigen irrespirablen Gase massgebend sein wird.

Für die Praxis ist es noch wichtig zu bemerken, welche ausserordentliche Bedeutung das Eintreten von Sekundärinfektion für den Gesamtverlauf der einzelnen Erkrankung hat. Auch wenn das Gift selbst nur ganz unbedeutende Schädigungen gesetzt haben sollte, so können diese ausreichen, um die Eingangspforte für eine tödliche Infektion zu schaffen. Insbesondere können auf diese Weise schwere Pneumonien und Vereiterungen der Speicheldrüse entstehen.

Es wird sich nunmehr um die Beantwortung der Frage handeln, welche Ansichten über den Mechanismus der Giftwirkung auf Grund der geschilderten Krankheitsbilder sich ergeben.

Bezüglich dieser Frage sei auf die allgemeinen Ausführungen auf Seite $8 \mathrm{ff}$. bingewiesen.

Besonders soll nur nochmals betont werden, dass - im Gegensatz zu der Auffassung von Weber - das beschriebene Krankheitsbild ganz deutlich auf eine Säurevergiftung hinweist. Die mikroskopischen Bilder der erkrankten Organe, zumal bei dem ersten Krankheitstyp, zeigen weitestgehende Uebereinstimmung mit den Befunden bei der durch den Krieg allgemein bekannt gewordenen Phosgenvergiftung, deren Charakter als Aetzvergiftung wohl kaum in Zweifel gezogen werden dürfte. Ferner spricht für die Annahme einer Säurevergiftung die von Weber bereits beschriebene Gelbfärbung der Atemwege bei Vergiftung mit sehr 
hohen Konzentrationen sowie die charakteristische sulzige Infiltration und Braunfärbung in der Umgebung der Injektionsstelle nach subkutaner Injektion.

Da die gleichen anatomischen Erscheinungen durch eine sehr grosse Reihe anderer chemischer Verbindungen verschiedenster Zusammensetzung hervorgerufen werden, welche ihrer chemischen Natur nach sämtlich als Säureabspalter aufgefasst werden müssen, so muss man diesen Schluss auch für das Dimethylsulfat in Anspruch nehmen.

\section{Zusammenfassung.}

1. Die funktionellen Veränderungen, welche die Alkylester der Mineralsäuren, der Kohlensäure, und der organischen Säuren, soweit sie in der vorliegenden Arbeit untersucht wurden, hervorrufen, bestehen in einer Verlangsamung und Verflachung der Atmung und in einer ausgesprochenen Blutdrucksenkung.

2. Daneben verursachen die Ester der Mineralsäuren bei ihrer Wirkung auf das Zentralnervensystem Krämpfe, die Ester der Kohlensäure und der organischen Säuren mehr oder weniger flüchtige Narkose.

3. Bei der Einatmung von Dimethyl-, Diäthylsulfat, Bromessigsäureester entstehen 3 verschiedene Krankheitstypen, für welche als Charakteristika gelten:

Erster Krankheitstypus: Primäres Lungenödem, sekundäre Herzschwäche, Stauungserscheinungen in allen Organen.

Zweiter Krankheitstypus; Primäre pseudomembranöse (meist absteigende) Pharyngitis, Laryngitis, Tracheitis und Bronchitis. Daneben besteht kein Oedem der Lunge.

Dritter Krankheitstypus: Allgemeine Gewebs- und Kapillargefässschädigung, wobei der Krankheitsverlauf als zunehmende Herzmuskelschwäche oder allgemeine toxische Kachexie imponiert. Voränderungen der Atmungsorgane fehlen.

In der Mehrzahl der Fälle entstehen Mischformen, welche in verschiedener Weise aus den 3 typischen Erkrankungen zusammengesetzt sind.

Die beschriebenen Krankheitstypen gelten allgemein für sämtliche Gaskampfstoffe; ebenso riefen eine Reihe von Stoffen, die jch in Rücksicht auf militärische und gewerbliche Verwendung untersucht habe, und die hier des Einzelnen nicht angeführt sein mögen, im Allgemeinen dieselben Symptome und Befunde hervor. Die oben gewählte Gruppierung passt sich auch den pathologisch-anatomischen und klinischen Erfahrungen am Menschen zwanglos ar. 